\title{
Glutamate Dehydrogenase Deficiency in Cerebellar Degenerations: Clinical, Biochemical and Molecular Genetic Aspects
}

\author{
Andreas Plaitakis, Panayiotis Flessas, Anastasia B. Natsiou and P. Shashidharan
}

\begin{abstract}
Glutamate dehydrogenase (GDH), an enzyme central to glutamate metabolism, is significantly reduced in patients with heterogenous neurological disorders characterized by multiple system atrophy (MSA) and predominant involvement of the cerebellum and its connections. In human brain, GDH exists in multiple isoforms differing in their isoelectric point and molecular mass. These are differentially reduced in quantity and altered in catalytic activity in patients with clinically distinct forms of MSA, thus suggesting that these GDH isoproteins are under different genetic control. Dysregulation of glutamate metabolism occurs in patients with GDH deficiency and is thought to mediate the disease's neurodegeneration via neuroexcitotoxic mechanisms. This possibility is supported by additional data showing that glutamate binding sites are significantly decreased in cerebellar tissue obtained at autopsy from MSA patients. At the molecular biological level, several cDNAs specific for human GDH have been isolated recently and cloned. Northern blot analysis of various human tissues, including brain, has revealed the presence of multiple GDH-specific mRNAs. In addition, multiple GDH-specific genes are present in humans and these data are consistent with the possibility that the various GDH isoproteins are encoded by different genes. These advances have laid the groundwork for characterizing the human GDH genes and their products in health and disease.
\end{abstract}

RÉSUMÉ: Déficit en glutamate déshydrogénase dans les dégénerescences cérébelleuses: aspects cliniques, biochimiques et de génétique moléculaire. La glutamate déshydrogénase (GDH), un enzyme important du métabolisme du glutamate, est diminuée de façon significative chez les patients souffrant de maladies neurologiques hétérogènes caractérisées par une atrophie multi-systémique (AMS) et une atteinte principalement localisée au cervelet et à ses voies de communication. Dans le cerveau humain, la GDH existe sous de multiples isoformes, qui diffèrent par leur point isoêlectrique et leur masse moléculaire, dont la quantité est diminuée différentiellement et dont l'activité catalytique est altérée, chez les patients qui ont des formes d'AMS cliniquement distinctes, suggérant que ces isoprotéines sont sous un contrôle génétique différent. Un dérèglement du métabolisme du glutamate survient chez les patients qui ont un déficit en GDH et l'on pense qu'il est à l'origine de la neurodégénérescence présente dans cette maladie via des mécanismes neuro-excitotoxiques. Des données additionnelles supportent cette possibilité: les sites de liaison du glutamate sont diminués de façon significative dans le tissu cérébelleux provenant d'autopsies de patients atteints d'AMS. Au niveau de la biologie moléculaire, plusieurs ADN complémentaires spécifiques pour la GDH humaine ont été isolés et clonés récemment. L'analyse par Northern blot de différents tissus humains incluant le cerveau, a révélé la présence de multiples ARN messagers spécifiques de la GDH. De plus, plusieurs gènes spécifiques à la GDH sont présents chez l'humain. Ces données supportent la possibilité que différentes isoprotéines de la GDH sont codées par des gènes différents. Ces travaux vont servir de base à la caractérisation des gènes de la GDH humaine et de leurs produits chez les sujets sains comme chez les sujets malades.

Can.J. Neurol. Sci. 1993; 20 (Suppl. 3): S109-S116

An impressive body of evidence has accumulated over the past decade suggesting that glutamate dysfunction may play a role in human degenerative disorders. Although much of this evidence is based on experimental work, of which relevance to human disorders remains largely speculative, detection of glutamate dehydrogenase (GDH) deficiency in patients with cerebellar disorders was one of the original observations that linked defective glutamate metabolism to neurodegenerative processes. 1.2
Clues to the presence of GDH abnormalities in human degenerative disorders were provided by the mode of action of the selective neurotoxin 3-acetylpyridine (3AP), an analog of nicotinamide. The systemic administration of the toxic agent to rats has resulted in selective lesioning of the inferior olives, olivocerebellar fibers, lower cranial nerve nuclei, and areas of the pons and nigra ${ }^{3}$ thus providing an experimental model for olivopontocerebellar atrophy (OPCA). ${ }^{1.2} 3 \mathrm{AP}$, acting as nicotinamide antagonist, is incorporated into the nicotinamide-ade-

From the Department of Neurology, Mount Sinai School of Medicine, New York (A.P., P.S.); University of Crete, School of Health Sciences, Iraklion, Crete, Greece (P.F., A.B.N.)

Reprint requests to: Andreas Plaitakis, M.D., Department of Neurology, Mount Sinai School of Medicine, One Gustave L. Levy Place, New York, N.Y., USA 10029 
nine dinucleotide phosphate (NADP) system forming substantial amounts of 3APADP in the brain of experimental animals. ${ }^{4}$ Inhibition of NADP-dependent oxidoreductases, by 3APADP, is thought to mediate the neurotoxic effects of $3 \mathrm{AP}$. It was then considered $^{1.2}$ that the neuronal systems that degenerate both in 3AP toxicity and in OPCA, may be selectively sensitive to the malfunction of one of these oxidoreductases.

To investigate this possibility, four NADP $(\mathrm{H})$-dependent dehydrogenases were measured in the cultured skin fibroblasts of a 19-year-old patient affected since age 11 by a progressive neurological disorder thought to represent a form of OPCA and characterized by juvenile parkinsonism, bulbar palsy, cerebellar ataxia, amyotrophy, and peripheral neuropathy. ${ }^{2}$ Results revealed that $\mathrm{GDH}$ activity was selectively reduced (to $22 \%$ of control) while other oxidoreductases such as glucose-6-phosphate dehydrogenase, isocitrate dehydrogenase and glutathione reductase were not significantly altered. ${ }^{2}$ GDH activity was also reduced in leukocytes isolated from peripheral blood of this patient.

Following these initial results, more extensive investigations were undertaken in our laboratory using primarily leukocytes isolated from peripheral blood. ${ }^{5-7}$ Eighty-eight patients with various types of degenerative neurological disorders affecting primarily the cerebellum and/or the basal ganglia were investigated. Twelve patients with slowly progressive multiple-system atrophic disorders not associated with autonomic dystrophy were found to have a partial deficiency of this enzyme (about $50 \%$ of control level). ${ }^{6}$ The age range of the GDH-deficient patients was 43 - 73 years (mean age, 64 years). Most of these patients showed a constellation of clinical findings (cerebellar, bulbar, oculomotor, corticospinal and extrapyramidal deficits) that was consistent with the diagnosis of OPCA, but others were atypical. Thus, GDH-deficient cases were encountered with predominantly extrapyramidal manifestations (atypical Parkinson's disease), bulbar palsy, motor neuronal degeneration or cerebellar ataxia with peripheral neuropathy. Five of the GDH deficient cases were sporadic and seven were familial with siblings but not parents or offsprings found to be neurologically affected, thus raising the possibility of recessive inheritance. ${ }^{6}$ Also, the possibility of dominant transmission with incomplete penetrance has been considered. ${ }^{7}$

With respect to other types of motor sytem disorders, GDH activity was found to be normal in patients affected by multisystem atrophy (MSA) with progressive autonomic failure. This clinically and radiologically distinct form of OPCA accounts for about $30 \%$ of the late onset sporadic cerebellar cases in our series. ${ }^{8}$ Leukocytic GDH was also normal in patients with Huntington's chorea, typical Parkinson's disease, amyotrophic lateral sclerosis, Charcot-Marie-Tooth disease, familial spastic paraplegia, Friedreich's ataxia and cerebello-olivary atrophy. However, patients with atypical. parkinsonism and progressive supranuclear palsy showed small but significant decreases in their leukocytic GDH (about $85 \%$ of control). ${ }^{9}$

GDH activity was normal in all but one dominant OPCA pedigrees studied by us. ${ }^{6}$ This was true for dominant OPCA kindreds linked to the HLA locus on the 6th chromosome (SchutHaymaker type), ${ }^{10}$ as well as for kindreds that do not map to this chromosomal locus (dominant OPCA with slowed saccades and spino-pontine atrophy). ${ }^{8}$ In these pedigrees, onset of disease occurred in early adult life (2nd - 3rd decade) and a complete penetrance of the mutant gene(s) has been established. In the single dominant pedigree in which GDH abnormalities were detected (see below), multisystemic involvement and childhood onset occurred. Affected patients evidenced a combination of cerebellar dysfunction; neuroretinal degeneration with blindness; peripheral neuropathy and bulbar, oculomotor, corticospinal, extrapyramidal, and anterior horn cell deficits. ${ }^{8}$

\section{Evidence for Selective Deficiency of GDH Isoforms in OPCA}

Although GDH in mammalian tissues is thought to be localized in the mitochondrial matrix and to be easily released in a soluble form by tissue homogenization," our investigations revealed evidence for the cellular presence of two distinct enzyme fractions: one that is bound to membranes (particulate $\mathrm{GDH}$ ) and another that is readily solubilized (soluble GDH), ${ }^{6,12}$ Studies on leukocyte homogenates isolated from OPCA patients revealed that the decrease in enzyme activity was limited to the membrane-bound component of the enzyme $e^{6.7 .13}$ thus raising the possibility that the two GDH activities are under different genetic control. Further studies on partially purified leukocytic preparations from control subjects showed that the two enzyme activities differed in their relative resistance to heat denaturation with the soluble enzyme being thermostable and the particulate GDH thermolabile. ${ }^{6}$

In view of these findings, heat-stable and heat-labile forms of GDH were measured in whole homogenates of leukocytes from patients and controls. Results revealed that the majority of patients with late onset sporadic OPCA associated with partial deficiency of total GDH activity, showed a selective reduction in the particulate (30\% of control) and heat-labile (about $10 \%$ of control) GDH activities. ${ }^{6.7 .14}$ Whole homogenates of cultured skin fibroblasts from such patients showed a selective reduction in the fraction of the enzyme that is activated by Triton X-100 ( $25 \%$ of control), ${ }^{13}$ thus further suggesting a defect in membrane-bound GDH. Deficiency, however, of the particulate, heat labile GDH has not been a consistent finding in OPCA patients. ${ }^{15.16}$ In the childhood onset dominant OPCA that is characterized by multi-systemic involvement and neuroretinal degeneration, a selective reduction in the soluble-thermostable GDH component has been found. ${ }^{17}$

Recently, GDH was purified to homogeneity from cerebellar tissue obtained at autopsy from normal controls and neurologic patients. ${ }^{17}$ Two dimensional nonequilibrium $\mathrm{pH}$ gradient gel electrophoresis revealed that the normal human brain enzyme consists of four major isoproteins which have been designated GDH 1,2,3 and 4 (Figure 1). ${ }^{17}$ These were found to be differentially expressed in the catalytically active soluble-thermostable and particulate-thermolabile fractions. In a patient with the childhood onset OPCA that is associated with retinal degeneration, GDH isoprotein 1, which in controls is more abundant than the other GDH isoproteins, was markedly and selectively reduced $^{17}$ (Figure 1).

Over the past 10 years, other investigators have also reported abnormalities in GDH activity in patients with cerebellar disorders and other multi-system atrophies. Yamaguchi et al. ${ }^{18}$ reported reduced GDH activities in five patients with late onset (40 - 50 years of age) sporadic OPCA of the Dejerine-Thomas type (79\% of control $p<0.001$ ) and two patients with late (age at onset: 45 - 70 years) cortical cerebellar atrophy (71\% of control; $\mathrm{p}<0.001)$. 


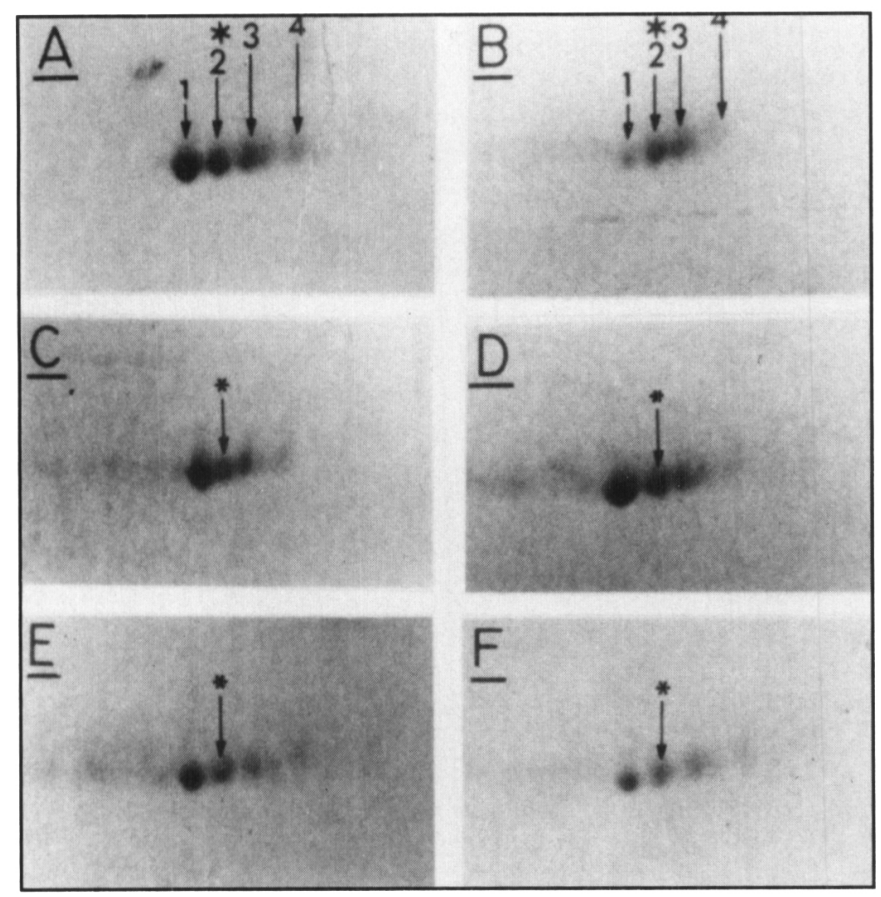

Figure I - Isoproteins of glutamate dehydrogenase purified to homogeneity from cerebellar autopsy tissue and analyzed by two dimensional nonequilibrium pH gradient electrophoresis. ${ }^{17} \mathrm{~A}$ : Neurologically normal subject who died of an acute myocardial infarction. B: Patient with non-HLA-linked form of dominant OPCA associated with retinal degeneration, slowed saccades, myoclonus, amyotrophy and peripheral neuropathy. $C$ and $F$ : Patient from the HLA-linked Schur-Haymaker kindred (non-GDH-deficient). D: Patient with amyotrophic lateral sclerosis. E: Patient with Parkinson's disease. Asterisk indicates ${ }^{35}$ S-labeled GDH synthesized by Hep $G 2$ cells. The labeled enzyme was immunoprecipitated from cell lysates and mixed (in trace amounts) with the purified human brain GDH. Its position, in relation to the 4 brain $G D H$ isoproteins (co-migration with the GDH isoprotein 2) was revealed by autoradiography of the slab gel. GDH isoprotein 1, which is more abundant than other GDH isoproteins in the cerebellum of normal and disease controls, is markedly reduced in the patient with the variant of dominant OPCA with retinal degeneration. Data are from ref ${ }^{\prime 7}$.

Duvoisin et al. ${ }^{19}$ described a partial deficiency in leukocytic GDH of nine OPCA patients out of 106 cases with different types of degenerative neurological disorders studied. The GDH deficient cases (ages 48 - 82 years; mean: 71 years) evidenced a late-onset sporadic degenerative disorder characterized by parkinsonian features, bulbar dysfunction (dysarthria, dysphagia), dysdiadochokinesia, intention tremor and oculomotor deficits (nystagmus, impaired pursuit, fixation instability and/or supranuclear palsy). All patients, but one, showed a type of gait disturbance that was thought to reflect a combination of extrapyramidal and cerebellar dysfunction. Corticospinal deficits were found in five and peripheral neuropathy in seven patients. ${ }^{20}$ The authors suggested that GDH deficiency is specifically associated with late onset sporadic OPCA. Additional studies, however, by Duvoisin et al. ${ }^{15}$ showed a much greater clinical heterogeneity than had been originally appreciated, necessitating the conclusion that partial GDH deficiency does not identify a particular type of multiple system atrophy.

Sorbi et al. ${ }^{21}$ found GDH activity to be partially deficient in platelets of four patients with non-dominant OPCA (ages 47 -
60 years) and two patients from a family affected by a dominantly inherited form of the disease. GDH assays were performed in whole platelet homogenates in the presence of Triton $X-100$ and with the presence or absence of the enzyme activator ADP. Stimulation of enzyme activity by ADP in these six patients was the same as it was in controls. However, in another dominant OPCA family, GDH activity was normal when assayed in the absence of ADP and Triton X-100 but partially deficient (about $50 \%$ of control) when assayed in the presence of these agents. Based on these findings, Sorbi et al. ${ }^{21}$ suggested that there may be at least two possible alterations of GDH in OPCA patients: one which decreases its catalytic activity and another which impairs its activation by ADP. However, the data presented by Sorbi et al. ${ }^{21}$ appear to suggest defective activation of the patient GDH by Triton X-100 and, as such, these results may be pertinent to our data showing impaired Triton $X-100$ activation of fibroblası GDH. ${ }^{13}$

In more recent studies, Sorbi et al. ${ }^{22}$ reported that, besides GDH, several milochondrial enzymes were reduced in patients with Friedreich's ataxia and non-dominant OPCA. However, in the non-dominant OPCA patients, the decrease in GDH activity (by about $50 \%, \mathrm{p}<0.01$ ) was greater than that of other mitochondrial enzymes (pyruvate dehydrogenase by $20 \%, \mathrm{p}<0.01$; valine dehydrogenase by $15 \%, \mathrm{p}<0.05$; succinate dehydrogenase by $10 \%, \mathrm{p}<0.05$ and citrate synthase by $7 \%, \mathrm{p}<0.05)$. In contrast to these results, patients with dominant OPCA showed a selective reduction in GDH activity only ( $50 \%$ of control).

Finocchiaro et al. ${ }^{23}$ determined GDH activity in leukocytes, fibroblasts and muscle mitochondria from 13 patients with dominantly inherited OPCA and from five patients with the recessive or sporadic forms of the disease. In patients with dominantly inherited disorders, GDH activity was significantly reduced in leukocytes ( $68 \%$ of control; $N=11$ ) and muscle mitochondria ( $46 \%$ of control; $N=4)$. GDH activity was also decreased $(65$ $85 \%$ of control) in leukocytes, fibroblasts and muscle mitochondria from the non-dominant OPCA patients. The reduction in GDH activity reported by Finocchiaro et al., ${ }^{23}$ was found when $\mathrm{NADPH}$, but not $\mathrm{NADH}$, was used together with ADP during assay.

Kanagaya et al. ${ }^{14}$ reported low GDH values in 12 patients classified as OPCA. Their ages ranged from $47-71$ years (mean: 56 years) and their clinical picture was characterized by a combination of extrapyramidal, cerebellar and corticospinal dysfunction. Some patients also exhibited abnormal eye movements and/or peripheral neuropathy. Total GDH activities were decreased to $77 \%$ of the mean control values: however, the heatlabile GDH component showed greater decreases ( $26 \%$ of control values) with no reduction in the thermostable enzyme fraction.

Aubby et al., ${ }^{16}$ studied leukocytic GDH of 158 patients with cerebellar and/or extrapyramidal disorders of various types and compared them to 71 normal and neurologic controls. Fourteen patients had GDH levels which were less than $50 \%$ of the mean control value. Of these five had ataxic disorders, three MSA, one juvenile Parkinson's disease, one dystonia-parkinsonism and four typical Parkinson's disease. The ataxic patients evidenced a late adult-onset (ages 54 - 82; mean: 66 years) progressive cerebellar syndrome associated with dementia, corticospinal deficits, oculomotor abnormalities and/or dystonic phenomena. All, but one were sporadic occurrences. The 
patients with multiple system atrophy (ages 43 - 54; mean: 51 years) evidenced parkinsonism, oculomotor abnormalities, peripheral neuropathy or autonomic dystrophy. The patient with juvenile parkinsonism had two sisters and one brother who were also similarly afflicted. The mean GDH activity of the various types of cerebellar and extrapyramidal disorders was significantly lower than that of controls, including patients with Parkinson's disease ( $85 \%$ of controls; $\mathrm{p}<0.01 ; \mathrm{N}=40$ ) and progressive supranuclear palsy $(72 \% ; \mathrm{p}<0.02 ; \mathrm{N}=8)$. Leukocyte GDH activity was fractionated by heat inactivation into thermolabile and thermostable components for the patients with total activity of less than $50 \%$ of the mean control value only, Results revealed significant reductions in both components.

The results of Aubby et al. ${ }^{16}$ are in general accord with those of our previous studies ${ }^{6.9}$ which showed that partial GDH deficiency occurs in clinically heterogenous disorders within the spectrum of multiple system atrophy. However, in contrast to our data ${ }^{6}$ as well as those of Duvoisin et al. ${ }^{19}$ and Kostic et al., ${ }^{24}$ Aubby et al. ${ }^{16}$ found GDH activity to be partially deficient in four patients with "typical" Parkinson's disease. However, all but one, of these "typical" Parkinson's patients experienced the onset of their disease during the second or third decade of life which is atypical for idiopathic Parkinson's disease and which has been considered as a criterion for classifying these cases under juvenile parkinsonism.

Orsi et al. ${ }^{25}$ found leukocytic GDH activity to be significantly decreased ( 2 SD below the mean control value) in 8 out of 29 patients (ages 12 - 78 years; mean: 45 years) affected by progressive ataxia. There was a significant correlation between GDH deficiency and the presence of additional neurologic features such as extrapyramidal signs, supranuclear palsy and peripheral neuropathy, thus also suggesting the multi-systemic nature of the disorder.

Kajiyama et al. ${ }^{26}$ recently reported three patients with cerebellar disorders (ages: 31,56 and 60 years) and one with juvenile Parkinsonism (age: 45 years) who had GDH activity that was lower than 2 SD from mean control value. The heat-labile GDH component was predominantly decreased. In these four patients the protein content of leukocyte GDH, as determined by a radioimmunoassay method, was significantly reduced with the ratio of GDH activity to protein content being the same in patients and controls. It was concluded that the reduced GDH activity was due (at least in part) to a decreased level of enzyme protein. The same group recently reported ${ }^{27}$ that 4 of 5 of their patients with decreased leukocytic GDH activity also showed reduced GDH activity in cultured skin fibroblasts. The viability of these cells was significantly decreased in the presence of toxic levels of L-glutamate as compared to that of controls, thus suggesting that abnormal sensitivity to glutamate toxicity may be involved in the neurodegeneration of GDH deficiency.

Kostic et al. ${ }^{24}$ measured GDH activity in platelets of 85 patients with degenerative cerebellar and/or basal ganglia disorders of various types. Significant reductions in GDH activity (38\% of control) were found in seven patients (ages $21-51$ years) with slowly progressive multi-system atrophy characterized by a combination of cerebellar, extrapyramidal and corticospinal deficits. Some patients also showed dementia, supranuclear oculomotor deficits, amyotrophy and peripheral neuropathy. Normal GDH activity was found in seven MSA patients with autonomic failure, 20 with Parkinson's disease, 10 with Huntington's chorea, five with essential tremor, five with Friedreich's ataxia, seven with Charcot-Marie-Tooth disease, 12 with dominantly inherited spinocerebellar degeneration and 10 with multiple sclerosis.

Iwatsuji et al. ${ }^{28}$ found that total and heat stable GDH activity in lymphocytes of normal controls increased significantly with aging. In contrast, patients with various types of primary system degeneration showed small but significant decreases in total and heat stable GDH activities as compared to age-matched controls. The heat-labile GDH decreased significantly in patients with OPCA only. Hence, these results raise important questions related to the expression of human GDH as a function of aging and invites further research to better understand the role of this enzyme in late onset neurodegenerations and the aging process.

Kaakola et al. ${ }^{29}$ measured GDH and hexosaminidase activity in 12 patients with dominant late onset ataxia, 15 patients with sporadic late onset ataxia and eight with alcoholic cerebellar degeneration. Results showed that the total GDH activity was significantly decreased in the sporadic $(p<0.01)$ and the dominant $(p<0.05)$ forms of ataxia with the heat labile fraction of $\mathrm{GDH}$ being reduced in the sporadic ataxia only. GDH activities were normal in the patients with alcoholic cerebellar degeneration. The serum hexosaminidase activities were also normal in all patients groups.

Abe et al. ${ }^{29 a}$ recently measured heat-stable and heat-labile forms of GDH in 22 patients with spinocerebellar degeneration evaluated ophthalmologically and electrophysiologically. A selective reduction in the heat-labile GDH $(20 \%$ of normal; $\mathrm{p}<$ 0.001 ) was found in seven patients, all of whom showed attenuated oscillatory potentials on electroretinography. In contrast, patients with normal oscillatory potentials had heat-labile GDH activity which was similar to that found in normal controls. These data provide additional evidence supporting an important role for GDH in human retina, particularly for the functions of neural elements responsible for the generation of oscillatory potentials. ${ }^{29 a}$

\section{Glutamate Metabolism in GDH-deficient OPCA}

To determine whether the above described GDH abnormalities result in metabolic alterations, the enzyme's substrates glutamate, $\alpha$-ketoglutarate and ammonia, were measured in the fasting plasma of GDH deficient patients and controls. Results revealed significant increases in plasma glutamate (by 137 . $150 \%$ ) and decreases in $\alpha$-ketoglutarate (by $21-22 \%)^{5,30}$ thus indicating a partial metabolic block at the oxidative deamination of glutamate to $\alpha$-ketoglutarate (GDH catalyzed reaction).

This possibility was further tested by performing glutamate loading tests $(60 \mathrm{mg} / \mathrm{kg}$ body weight taken orally after overnight fasting). Results revealed that glutamate levels increased excessively in the plasma of the patients as compared to controls. This was associated with proportional increases in the plasma aspartate levels, thus indicating an intact transamination pathway. Similar observations have been made by other investigators in patients with dominant and sporadic OPCA associated with reduced GDH activity. ${ }^{23.27}$ In a recent report, Sawada et al. ${ }^{31}$ described that glutamate levels were elevated in the plasma and urine and decreased in the CSF of a patient with spinocerebellar degeneration. In addition, the levels of cysteine were elevated only in the urine. 


\section{Biochemical Changes in Brain}

Studies on brain tissue obtained at autopsy revealed that glutamate levels were reduced in the cerebellum both in the childhood-onset dominant OPCA (to $25 \%$ of control) ${ }^{17}$ as well as the late-onset sporadic disease (to $40 \%$ of control) (Plaitakis, unpublished data). The levels of other amino acids were not significantly altered with the exception of aspartate which was decreased (to $40 \%$ of control) in the dominant case. ${ }^{17}$

\section{Pathophysiology of Brain Topographic Lesions}

Because GDH plays a central role in the metabolism of the neuroexcitatory amino acid glutamate, detection of this enzyme deficiency has uncovered a direct link between a genetic molecular defect and neuroexcitotoxic mechanisms. As such, the study of GDH-deficient OPCA, a disorder characterized by classic system atrophy, has permitted us to gain insights into the mechanisms involved in the phenomenon of neuronal degeneration and the factors determining the topographically characteristic brain lesions that occur in these disorders.

Immunocytochemical investigations by Aoki et al. ${ }^{32}$ have shown that, under conditions expected to reveal the membranebound GDH, enzyme immunoreactivity was markedly enriched in CNS regions that receive putative glutamatergic innervation. In these regions GDH seems to be localized primarily in astrocytic processes associated with glutamatergic terminals. These astrocytes are thought to be responsible for removing synaptic glutamate by an energy-dependent high affinity uptake system. ${ }^{33.34}$ Glutamate, taken up by these cells, seems to be catabolized mainly via $\mathrm{GDH}^{35}$ to $\alpha$-ketoglutarate which may be transported back to the nerve terminals to serve as precursor of transmitter glutamate. ${ }^{36}$

A defect in GDH may therefore impair the ability of the glial cells to metabolize glutamate, leading to excessive accumulation of the transmitter at the synaptic cleft and neuroexcitotoxic degeneration of post-synaptic neurons. The study of morphological changes of brain in living patients with the use of neuroimaging techniques, ${ }^{37}$ as well as the examination of brain at autopsy, ${ }^{38}$ has indeed revealed a characteristic topography of brain lesions that can be attributed to degeneration of CNS structures which receive putative glutamatergic innervation (Figure 2) and which are rich in GDH activity. ${ }^{32}$

How this neuroexcitotoxic hypothesis can be reconciled with data obtained on autopsy brain tissue which revealed significant decreases in the tissue content of glutamate ${ }^{17}$ remains unclear. It should be noted, however, that glutamate measured in brain samples represents primarily the intracellular compartment (the extracellular levels are very low). Decreased glutamate degradation by synaptic astrocytes due to GDH deficiency may disrupt the recycling of the transmitter (due to decreased supply of precursors to the nerve terminals) with resultant depletion of the intracellular glutamate content.

Some authors have suggested that GDH in the CNS is involved primarily in the synthesis of glutamate from glucose ${ }^{39}$ rather than in the breakdown of the amino acid. If this is so, malfunction of GDH is expected to lead to reduced formation of glutamate and this could account for the depletion of this amino acid occurring in the brain of patients with GDH deficiency. These considerations, taken along with recent observations indicating that glutamate receptors may have a neurotrophic function promoting normal growth and development of cerebellar

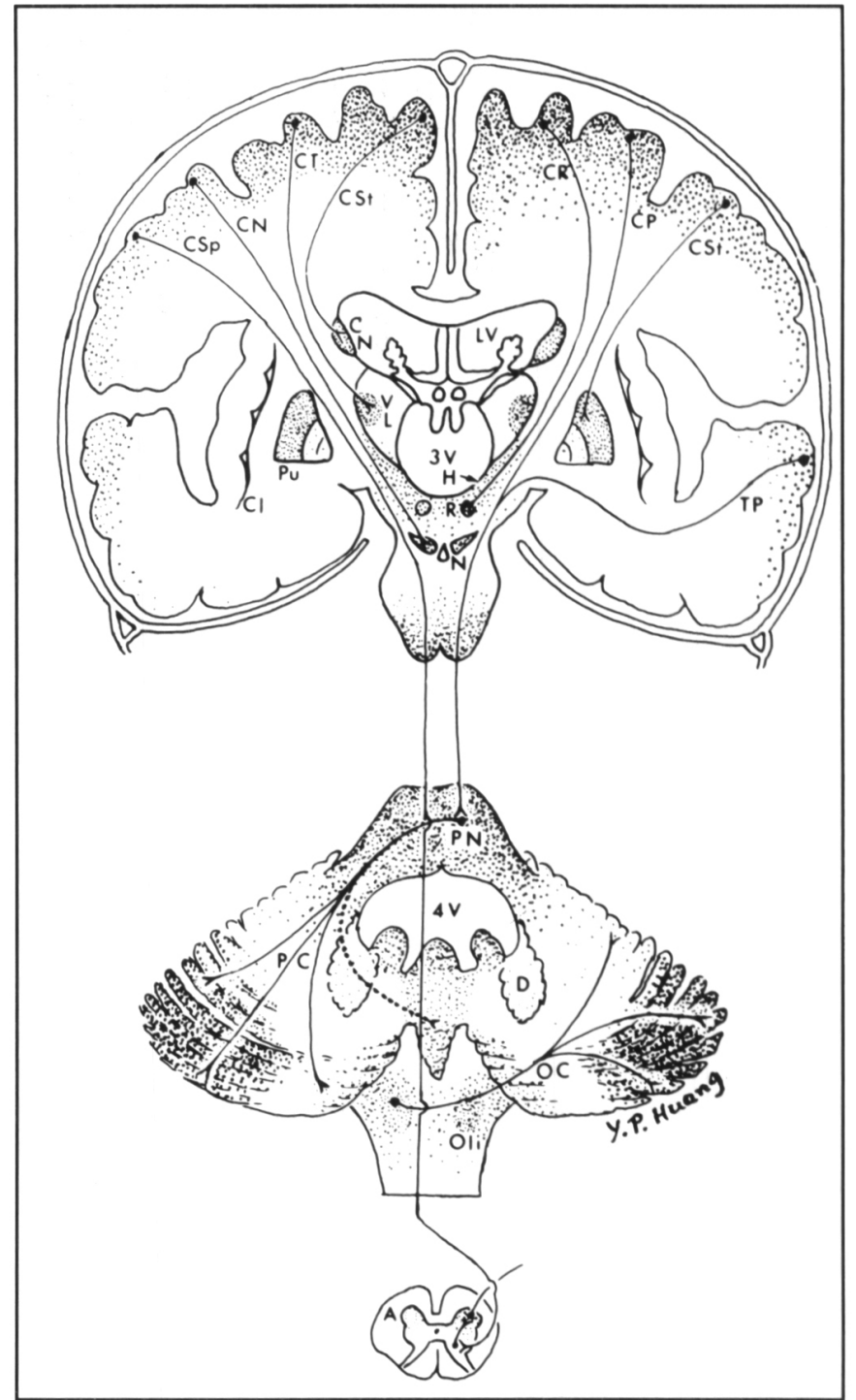

Figure 2 - Diagram showing putative glutamatergic fiber systems of the brain in relation to morphologic alterations occurring in GDH deficient OPCA. Dotted areas are thought to receive glutamatergic innervation as described by Huang and Plaitakis. ${ }^{37}$ These glutamatergic receptive regions correspond to rat brain areas shown by Aoki et al. 32 to be particularly rich in GDH immuonreacrivity. Degenerative changes involving many of these regions have been revealed by $C T$ and $M R I$ in GDH deficient OPCA patients. ${ }^{37}$ Putative glutamatergic tracks labeled: $C N$ : Corticonigral: $C P$ : Corticopontine; CR: Corticorubral; Cst: Corticostriatal; CT: Corticothalamic; $O C$ : Olivocerebellar; and $P C$ : Pontocerebellar: This Figure is from reference ${ }^{38}$.

cells, ${ }^{40.41}$ raise the possibility that decreased synthesis of glutamate in nerve tissue may lead to premature degeneration by attenuating a neurotrophic support essential for nerve cells. Further studies are needed to address these exciting issues which may have implications for understanding human neurodegenerations in general.

\section{L-glutamate Receptors in OPCA}

If the above neuroexcitotoxic hypothesis for the pathogenesis of OPCA is correct, one would expect to find in this disorder a 
selective disappearance of glutamatergic post-synaptic neurons bearing glutamate receptors. To test this possibility, L-glutamate binding was measured in cerebellar cortical tissue of four OPCA patients. Two of these were associated with GDH deficiency as described above whereas the other two were members of the Schut-Haymaker dominant OPCA kindred (non-GDH deficient). ${ }^{10}$ Results revealed the L-glutamate binding was significantly decreased (to $30 \%$ of control) in all these patients without a change in the affinities and the pharmacologic properties of the binding sites. ${ }^{42}$ Additional autoradiographic studies ${ }^{43}$ showed significant decreases in quisqualate-sensitive L-glutamate binding in the molecular layer of the cerebellum. In some patients, particularly those associated with GDH abnormalities, L-glutamate binding was decreased in the granule layer as well. Similar results have also been obtained in these patients by Albin et al ${ }^{44}$ who reported significant reductions in quisqualate and non-quisqualate/non-NMDA glutamate binding in the molecular layer, and in the NMDA receptor in the granule layer. Moreover, these authors ${ }^{45}$ showed that the ionotropic and metabotropic quisqualate receptors were significantly decreased in the OPCA cerebellum.

\section{Genetics of GDH-Deficient Disorders}

In studies which evaluated a rather large number of patients suffering from degenerative neurological disorders of various types, GDH deficiency was found in $20-50 \%$ of cases in which a clinical diagnosis of OPCA appeared tenable. ${ }^{6.16,19.24}$ At present, there are no reliable clinical criteria that can distinguish between the GDH-deficient and the GDH-normal cases, although the frequent association of neuropathy ${ }^{20}$ in the former, and of progressive autonomic failure with orthostatic hypotension in the latter ${ }^{6.24}$ have been suggested.

The majority of cases with partial GDH deficiency were sporadic occurrences. However, others had similarly affected siblings (but no parents nor offsprings) thus suggesting a possible recessive inheritance pattern in these cases. In seven pedigrees of our series, affected members were all males; a genetic transmission pattern compatible with an X-linked recessive disorder in these families. It is of particular interest in this regard that an intronless GDH-specific gene has been assigned to human chromosome $X .^{46.47}$ We have recently isolated and characterized a cDNA from human retinal cDNA library (Shashidharan and Plaitakis, unpublished data) the sequence of which was found to be identical to an intronless gene isolated from a human genomic library (Michaelidis et al., unpublished data).

Several GDH-deficient cases have been reported by Italian investigators in which the mode of genetic inheritance was clearly dominant. ${ }^{21.23 .25}$ In these, as in our dominant case, onset of the disease was often in childhood. ${ }^{21,25}$ Kaakola et al., ${ }^{29}$ however, reported reduced GDH activity in a group of 12 Finnish patients with late onset dominant cerebellar degeneration but only three patients had GDH activities which were below the lower control values. All but one of our dominant OPCA kindreds ( 16 dominant OPCA cases from five families of our series) had normal GDH activity in their leukocytes. ${ }^{6.7}$ Also, $\mathrm{GDH}$ activity was normal in the brain of patients from the Schut-Haymaker dominant OPCA pedigree who came to autopsy. ${ }^{17}$ Aubby et al. ${ }^{16}$ also found no GDH-deficient cases among 19 patients from 15 families with autosomal dominant cerebellar ataxia. Similar conclusions were drawn by Kostic et al..$^{24}$ who reported normal GDH activity in all five patients (from three families) with dominant OPCA.

\section{Molecular Genetics of Human GDH}

Substantial progress has been made recently in understanding the genetics of human GDH following the cloning of cDNAs encoding for human GDH. ${ }^{49.50}$ This has permitted the detection of four different-sized GDH-specific mRNAs in human tissues and has provided evidence for the presence of a multigene family (probably $3-5$ genes) in the human. ${ }^{50}$ Two chromosomal loci have been demonstrated for human GDH: one mapping on the long arm of chromosome 10 (bands: q21.1 - 21.2 or q23) and another, lacking introns, on the long arm of chromosome $X$ (bands: q26 - 28 or q24). ${ }^{46.47}$ Jung et al. ${ }^{46}$ pointed out that the GDH locus in human chromosome 10 is near the rare fragile site, folic acid sensitive, fra (10)(q23.3) ${ }^{46}$ An additional DNA band has also been detected showing no concordance with either chromosome 10 or $X^{477}$ its chromosomal localization is currently under investigation. Recent studies on genomic DNA have confirmed the presence of two GDH-specific split genes showing marked differences in the size of their introns. ${ }^{52}$

As described above, partial GDH deficiency occurs in clinically and genetically heterogeneous forms of OPCA and other multi-system atrophies. It is presently unclear whether the decrease in enzyme activity results from mutation(s) of the GDH gene(s) or reflect mitochondrial damage. ${ }^{38}$ Defective mitochondrial function has been recently suggested for certain primary degenerations such as Parkinson's disease. Therefore, mitochondrial damage may account for the small reductions in GDH activity shown in patients with atypical parkinsonism and progressive supranuclear palsy. ${ }^{9}$ In disorders, however, in which selective defects in GDH isoforms have been shown, GDH deficiency may constitute the primary genetic defect.

To establish whether GDH deficiency is primary to the disease process, demonstration of abnormal amino acid sequence of a GDH isoprotein and/or abnormal DNA sequence of a GDHspecific gene is required. The presently available protein chemistry techniques permit the characterization of GDH at the primary structure level. Mutations that can be uncovered by these techniques are those which alter either the quantity (Figure 1) or the electrophoretic mobility of the different GDH isoproteins. At present, this method is suitable for studying brain autopsy material. Application of these techniques in more readily available non-neural tissues such as leukocytes, platelets or fibroblasts remains problematic because these cells are usually not available in amounts sufficient for purification and analysis of GDH. However, the use of the Western blotting technique in conjunction with the electrophoretic analysis method, may permit the visualization of the different GDH isoproteins in crude tissue extracts thus providing a suitable method for testing for GDH polymorphism in the neurologic patients.

\section{The Use of the PCR Method for Detection of GDH Gene(s) Mutations}

Sequencing of DNA has been markedly facilitated by the recent development of a DNA amplification method which uses the polymerase chain reaction (PCR). ${ }^{53}$ This is an in vitro method of nucleic acid synthesis by which a particular segment of DNA can be specifically replicated in amounts sufficient to permit direct sequencing. The procedure involves the use of two 
oligonucleotide primers constructed on the basis of known DNA sequences that flank the fragment of DNA which is to be amplified. During the PCR, the target DNA strands are separated by heat denaturation which then allows the primers to anneal to opposite strands of the DNA template at the sites of complementary sequences. A heat-stable DNA polymerase is then used to synthesize (extension) the DNA segment that lies between the two primers replicating faithfully the DNA used as template. Repeated cycles of heat denaturation-annealing-extension are used for producing millions of molecules from a few used as templates. Since the extension products themselves are also complementary to and capable of binding primers, successive cycles of amplification essentially double the amount of the target DNA synthesized in the previous cycle. The result is an exponential accumulation of the specific target fragment, approximately $2^{n}$, where $n$ is the number of cycles of amplification performed. ${ }^{54}$ An important feature of this procedure is that the original template DNA is double stranded and the amplification products are also double stranded DNA fragments which include the sequences between the two PCR primers. ${ }^{55}$ Several kinds of template DNA, such as genomic DNA and cDNA, are suitable for PCR amplification.

Amplification of genomic DNA with the use of the PCR method requires knowledge of the organization and structure of the gene to be amplified. It is well known that most mammalian genes are composed of coding regions that are called exons which are interrupted by non-coding intervening sequences which are called introns. The size of such introns can be quite large, spanning several kilobases. Amplification of an entire intron-exon-containing gene (also called split gene) by one PCR reaction is not possible for most mammalian genes because they usually span many kilobases. The maximum size of DNA that can be amplified by PCR is usually not larger than 2 kilobases. As such, a common practice has been the amplification of each exon using primers that correspond to adjacent intron sequences.

As detailed above, there is evidence for the existence of several GDH-specific genes in the human. The organization and structure of one split gene that encodes for the published liver cDNA has been characterized. ${ }^{56}$ The gene is composed of 13 exons interrupted by introns of variable length. The sequence of these introns at the exon/intron junctions have been determined. Based on this information, we have synthesized sets of PCR primers that correspond to intron sequences that flank each of these exons. Using human genomic DNA we have been able to amplify most of these exons. Uneven concentrations of primers were also used to synthesize single stranded DNA for performing direct sequencing.

Results obtained thus far revealed that the sequence of the amplified GDH gene exons is in accord with that of the corresponding regions of the published cDNA. ${ }^{50}$ In addition, amplification of exons corresponding to the second GDH-specific split gene, ${ }^{52}$ was also detected. Additional studies are now in progress which involve amplification and direct sequencing of exons using genomic DNA from patients with reduced GDH activity.

\section{ACKNOWLEDGEMENTS}

This work was supported by NIH grants NS-16871 and RR00071, the Mount Sinai General Clinical Research Center. We are indebted to
Dr. DD Clarke for reviewing this manuscript and to Betsy Chalfin for editorial assistance in the preparation of this manuscript. We are also thankful to many colleagues who made these investigations possible by referring their patients to us.

\section{REFERENCES}

1. Plaitakis A, Nicklas WJ, Desnick RJ. Glutamate dehydrogenase deficiency in three patients with spinocerebellar ataxia: a new enzymatic defect? Trans Am Neurol Assoc 1979; 104: 54-57.

2. Plaitakis A, Nicklas WJ, Desnick RJ. Glutamate dehydrogenase deficiency in three patients with spinocerebellar syndrome. Ann Neurol 1980; 7: 297-303

3. Desclin JC, Escubi J. Effects of 3-acetylpyridine on the central nervous system of the rat as demonstrated by silver methods. Brain Res 1974; 77: 349-364.

4. Herken H. Functional disorders of the brain induced by synthesis of nucleotides containing 3-acetylpyridine. Z Klin Chem 1968; 6: 635-367.

5. Plaitakis A, Berl S. Oral glutamate loading in disorders with spinocerebellar and extrapyramidal involvement: effect on plasma glutamate, aspartate and taurine. J Neural Transm 1983; (Suppl 19): 65-74.

6. Plaitakis A, Berl S, Yahr, M.D. Neurological disorders associated with deficiency of glutamate dehydrogenase. Ann Neurol 1984; 15: 144-153.

7. Plaitakis A. Abnormal metabolism of neuroexcitatory amino acids in olivopontocerebellar atrophy. In: Duvoisin RC. Plaitakis A, eds. The Olivopontocerebellar Atrophies. Adv Neurol. New York: Raven Press 1984; 41: 225-243.

8. Plaitakis A. Classification and epidemiology of cerebellar degeneration. In: Plaitakis A, ed. Cerebellar Degenerations: Clinical Neurobiology. Boston: Kluwer Academic Publishers 1992: 185204.

9. Plaitakis A, Yahr MD. Abnormalities in GDH and glutamate metabolism in atypical Parkinson's disease and progressive supranuclear palsy. Neurology 1986; 35 (Suppl 1): 110.

10. Haines JL, Schut LJ, Weikamp LR, et al. Spinocerebellar ataxia in a large kindred: age at onset, reproduction, and genetic linkage studies. Neurology 1984; 34: 1542-1548.

11. Smith EL, Austen BM, Blumenthal KM, Nyc JF. Glutamate dehydrogenases. In: Boyer, ed. The Enzymes. New York: Academic Press 1975; 293-367.

12. Colon A, Plaitakis A, Perakis A, et al. Purification and characterization of a soluble and a particulate glutamate dehydrogenase from rat brain. J Neurochem 1986; 46: 1811-1819.

13. Plaitakis A. Biochemistry of recessive olivopontocerebellar atrophy. Ital J Neurol Sci 1984; (Suppl 4): 65-73.

14. Konagaya Y, Konagaya M, Takayanagi T. Glutamate dehydrogenase and its isozyme activity in olivopontocerebellar atrophy. J Neurol Sci 1986; 74: 231-236.

15. Duvoisin RC, Nicklas WJ, Ritchie V, et al. Low leukocyte glutamate dehydrogenase activity does not correlate with a particular type of multiple system atrophy. J Neurol Neurosurg Psychiatry 1988; 51: 1508-1511.

16. Aubby D, Saggu HK, Jenner P, et al. Leukocyte glutamate dehydrogenase activity in patients with degenerative neurological disorders. J Neurol Neurosurg Psychiatry 1988; 51: 893-902.

17. Hussain MM, Zannis V, Plaitakis A. Characterization of glutamate dehydrogenase isoproteins purified from the cerebellum of normal subjects and patients with degenerative neurological disorders, and from human neoplastic cell lines. J Biol Chem 1989; 264: 20730-20735.

18. Yamaguchi T, Hayashi K, Murakami H, et al. Glutamate dehydrogenase deficiency in spinocerebellar degenerations. Neurochem Res 1982; 7: 627-636.

19. Duvoisin RC, Chokroverty S, Lepore F, Nicklas WJ. Glutamate dehydrogenase deficiency in patients with olivopontocerebellar atrophy. Neurology 1983; 33: 1322-1326.

20. Chokroverty S, Duvoisin RC, Sacheo R, et al. Neurophysiologic study of olivopontocerebellar atrophy with or without glutamate dehydrogenase deficiency. Neurology 1985; 35: 652-659. 
21. Sorbi S, Tonini S, Giannini E, Piacetini S, et al. Abnormal platelet glutamate dehydrogenase activity and activation in dominant and non-dominant olivopontocerebellar atrophy. Ann Neurol 1986; 19: 239-245.

22. Sorbi S, Piacetini S, Fani C, et al. Abnormalities of mitochondrial enzymes in hereditary ataxias. Acta Neurol Scand 1989; 80: 103110.

23. Finocchiaro G, Taroni F, DI Donato S. Glutamate dehydrogenase in olivopontocerebellar atrophies: leukocytes, fibroblasts, and muscle mitochondria. Neurology 1986; 36: 550-553.

24. Kostic VS, Mojsilivic LJ, Stojanovic M. Degenerative neurological disorders associated with deficiency of glutamate dehydrogenase. J Neurol 1989; 236: 101-114.

25. Orsi L, Bertolotto A, Bringolio $F$, et al. Glutamate dehydrogenase (GDH) deficiency in different types of progressive hereditary cerebellar ataxia. Acta Neurol Scand 1988; 78: 394-400.

26. Kajiyama K, Ueno $S$, Tatsumi $T$, et al. Decreased glutamate dehydrogenase protein in spinocerebellar degeneration. J Neurol Neurosurg Psychiatry 1988; 51: 1078-1080.

27. Tatsumi C, Yorifuji S, Takahashi M, Tarui S. Decreased viability of skin fibroblasts from patients with glutamate dehydrogenase deficiency. Neurology 1989; 39: 541-452.

28. Iwattsuji K, Nakamura S, Kameyama M. Lymphocyte glutamate dehydrogenase activity in normal aging and neurological diseases. Gerontology 1989; 35: 218-224.

29. Kaakola S, Marnela K-M, Oja SS, et al. Leukocyte glutamate dehydrogenase and CSF amino acids in late onset ataxias. Acta Neurol Scand 1990; 82: 225-229.

29a. Abe T, Ishiguro S-I, Saito H, et al. Partially deficient glutamate dehydrogenase activity and attenuated oscillatory potentials in patients with spinocerebellar degeneration. Invest Ophthalmol Vis Sci 1992; 33: 447-452.

30. Plaitakis A, Berl S, Yahr MD. Abnormal glutamate metabolism in adult-onset degenerative neurological disorder. Science 1982; 216: 193-196.

31. Sawada H, Seriu N, Udaka F, et al. Cerebellar ataxia with glutamic aciduria. Acta Neurol Scand 1991; 84: 70-72.

32. Aoki C, Milner TA, Rex Sheu K-FR, et al. Regional distribution of astrocytes with intense immunoreactivity for glutamate dehydrogenase in rat brain: implications for neuron-glia interactions in glutamate transmission. J Neurosci 1987; 7: 2214-2231.

33. Fonnum F. Glutamate: a neurotransmitter in mammalian brain. $J$ Neurochem 1984; 42: 1-11.

34. Balcar VJ, Borg J, Mandel P. High affinity uptake of L-glutamate and L-aspartate by glial cells. J Neurochem 1977; 27-28.

35. Hertz L, Schousboe A. Metabolism of glutamate and glutamine in neurons and astrocytes in primary cultures. In: Kvamme E, ed. Glutamine and Glutamate in Mammals. Boca Raton, FL, CRC Press 1988; II: 39-55.

36. Shank RP, Aprison MH. Glutamate as neurotransmitter. In: Kvamme E, ed. Glutamine and Glutamate in Mammals. Boca Raton, FL, CRC Press 1988; II: 3-19.

37. Huang YP, Plaitakis A. Morphological changes in olivopontocerebellar atrophy in computed tomography and comments on its pathogenesis. In: Duvoisin RC, Plaitakis A, eds. The Olivopontocerebellar Atrophies. Adv Neurol. New York: Raven Press 1984; 41: 39-81.

38. Plaitakis A. Olivopontocerebellar atrophy with glutamate dehydrogenase deficiency. In: JMBV de Jong, ed. Handbook of Clinical Neurology, Hereditary Neuropathies and Spinocerebellar Atrophies. Amsterdam: Elsevier Science Publishers 1990; 16(60): $551-568$.
39. Chee PY, Dahl LJ, Fahien A. The purification and properties of rat brain glutamate dehydrogenase. J Neurochem 1979; 33: 53-60).

40. Pearce IA, Cambray-Deakin MA, Burgyne RD. Glutamate acting on NMDA receptors stimulates neurite outgrowth from cerebellar granule cells. FEBS Lett 1987; 233: 143-147.

41. Balazs R, Hack N, Jorgensen OS. Stimulation of the N-methyl-Daspartate receptor has a trophic effect on differentiating cerebellar granule cells. Neurosci Lett 1988; 87: 80-86.

42. Tsiotos P, Plaitakis A, Mitsakos A, et al. L-glutamate binding sites of normal and atrophic human cerebellum. Brain Res 1989; 481: 87-96.

43. Hatziefthimiou A, Mitsakos A, Mitsaki E, et al. Quantitative autoradiographic study of L-glutamate binding studies in normal and atrophic human cerebellum. J Neurosci Res 1991; 28: 367 375.

44. Albin RL, Gilman S. Autoradiographic localization of inhibitory and excitatory amino acid transmitter receptors in human normal and olivopontocerebellar atrophy cerebellum. Brain Res 1990; 522: $37-45$.

45. Macowiec RL, Albin RL, Cha JJ, et al. Two types of quisqualate receptor are reduced in human OPCA cerebellar cortex. Brain Res 1990; 552: 37-45.

46. Jung KY, Warter S, Rumpler Y. Assignment of the GDH loci to human chromosomes 10q23 and Xq24 by in situ hybridization. Ann Genet 1989; 32: 109-110.

47. Anagnou NP, Senanez H, Modi SJ, et al. Chromosomal mapping of the human glutamate dehydrogenase (GLUD) genes to chromosomes 10 q21.1-21.2 and X q26-28. Am Soc Hum Genet 1989; 45: A170.

48. Rich SS, Wilkie PJ, Schut LJ, et al. Spinocerebellar ataxia: localization of an autosomal dominant locus between two markers on human chromosome 6. Am J Hum Genet 1987; 41: 524-531.

49. Banner C, Silverman S, Thomas JW, et al. Isolation of a human cDNA clone for glutamate dehydrogenase. J Neurochem 1987; 49: $246-252$.

50. Maurothalassitis G, Tzimagiorgis G, Mitsialis A, et al. Isolation and characterization of CDNA clones encoding human liver glutamate dehydrogenase: evidence for a small gene family. Proc Natl Acad Sci USA 1988; 85: 3494-3498.

51. Hanauer A, Mattei MG, Mandel JL. Presence of a tag I polymorphism in the human glutamate dehydrogenase (GLUD) gene on chomosome 10. Nucleic Acid Res 1987; 15: 6308.

52. Amuro N, Goto $\mathrm{Y}$, Okazaki $\mathrm{T}$. Isolation and characterization of two distinct genes for human glutamate dehydrogenase. Biochem Biophys Acta 1990; 1049: 216-218.

53. Saiki RK, Gelfard DH, Stoffel SV, et al. Primer-directed enzymatic amplification of DNA with a thermostable DNA polymerase. Science 1988; 239: 487-491.

54. Randall K, Saiki RK. Amplification of genomic DNA. In: Innis MA, Gelfand DH, Sninsky JJ, White TJ, eds. PCR Protocols. A Guide to Methods and Applications. San Diego: Academic Press 1990; 13-20.

55. Gibbs RA. DNA Amplification by the polymerase chain reaction. Anal Chem 1990; 62: 1202-1214.

56. Michaelidis TM, Tzimagiorgis G, Moschonas NK, Papamatheakis J. The human glutamate dehydrogenase gene family: Gene organization and structural characterization. Genomics, 1993 (in press). 